\title{
Measuring the Extent and Width of Internal Energy Deposition in Ion Activation Using Nanocalorimetry
}

\author{
William A. Donald and Evan R. Williams \\ Department of Chemistry, University of California-Berkeley, Berkeley, California, USA
}

\begin{abstract}
The recombination energies resulting from electron capture by a positive ion can be accurately measured using hydrated ion nanocalorimetry in which the internal energy deposition is obtained from the number of water molecules lost from the reduced cluster. The width of the product ion distribution in these experiments is predominantly attributable to the distribution of energy that partitions into the translational and rotational modes of the water molecules that are lost. These results are consistent with a singular value for the recombination energy. For large clusters, the width of the energy distribution is consistent with rapid energy partitioning into internal vibrational modes. For some smaller clusters with high recombination energies, the measured product ion distribution is narrower than that calculated with a statistical model. These results indicate that initial water molecule loss occurs on the time scale of, or faster than energy randomization. This could be due to inherently slow internal conversion or it could be due to a multi-step process, such as initial ion-electron pair formation followed by reduction of the ion in the cluster. These results provide additional evidence for the accuracy with which condensed phase thermochemical values can be deduced from gaseous nanocalorimetry experiments. (J Am Soc Mass Spectrom 2010, 21, 615-625) @ 2010 American Society for Mass Spectrometry
\end{abstract}

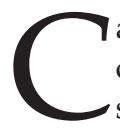
apture of a low-energy electron by a multiply charged peptide or protein can result in extensive and relatively non-selective fragmentation of the amide backbone, from which information about the amino acid sequence [1-3], post-translational modification sites [3-5], and higher-order structure $[2,6,7]$ can be obtained. Similar fragmentation can be obtained by transferring an electron to the peptide or protein cation from a molecular anion [8] or from atoms $[9,10]$. These methods are being applied to both "bottom up" and "top-down" approaches to protein analysis.

An important parameter in understanding how activated ions fragment is the extent of internal energy that is deposited in the activation step. For electron capture dissociation (ECD) [1-6], the recombination energy (RE) resulting from capture of an electron from a multiply charged peptide or protein has been estimated to be between 4 and $7 \mathrm{eV}[1,11,12]$, based in part on known or estimated thermochemical data. Dissociation may occur from excited electronic states or from ground states $[13,14]$, so that the internal energy deposited into an ion may not be a singular value.

Some ions have been used as chemical thermometers to measure internal energy deposition [15-18]. For example, dissociation of the molecular ion of $n$-butylbenzene

Address reprint requests to Professor E. R. Williams, Department of Chemistry, University of California-Berkeley, B42 Hildebrand Hall, Berkeley, CA 94720-1460, USA. E-mail: Williams@cchem.berkeley.edu results in formation of product ions at $m / z$ 91 and 92, the ratio of which provides information about the average internal energy of the activated precursor [15, 16]. Ions that dissociate via sequential fragmentation and that have known threshold dissociation energies and similar dissociation entropies have also been used [18-20]. From the abundance of the product ions, information about both the average energy and the width of the energy deposition can be inferred.

An alternative approach to measuring internal energy deposition uses nanometer-sized hydrated ions as "nanocalorimeters," to precisely measure the amount of energy that is deposited into these ions upon activation [21-30]. When these ions are activated, they sequentially lose water molecules. Under conditions where these ions are stored at low pressure and at low temperature, the internal energy deposition upon activation can be obtained from the number of water molecules lost, i.e., the energy deposited into the ions goes into breaking the interactions between the departing water molecules and the rest of the cluster, and into translational, rotational, and vibrational modes of the products. The number of water molecules that are lost upon electron capture (EC) by large hydrated ions can be high. For example, $\mathrm{Ca}\left(\mathrm{H}_{2} \mathrm{O}\right)_{32}^{2+}$ loses 10 or 11 water molecules and $\mathrm{Ru}\left(\mathrm{NH}_{3}\right)_{6}\left(\mathrm{H}_{2} \mathrm{O}\right)_{55}^{3+}$ loses 17 to 19 water molecules upon EC $[22,24]$. From the sum of the threshold water molecule binding energies and the amount of energy that is partitioned into translational, 
rotational, and vibrational modes of the products for each lost water molecule, the internal energy deposited into the ion can be obtained. Because the EC energy deposition is much faster than the timescale of the experiments, the energy resulting from solvent reorganization to accommodate the change in the oxidation state of the cluster is reflected in the number of water molecules that are lost from the reduced clusters. Thus, the RE values obtained from this nanocalorimetry method correspond to adiabatic ionization energies of the reduced precursors. This method has been used to measure the internal energy deposited with ECD of a diprotonated peptide [29], and has also been used to obtain absolute reduction energies of hydrated ions that can be related to solution-phase reduction potentials $[22,25,27]$. Using different nanocalorimetry based methods, three values for the absolute standard hydrogen electrode (SHE) potential that all agree within $5 \%$ of each other $(+4.05,+4.11$, and $+4.21 \mathrm{~V})$ were obtained $[22,25,27]$. These values can also be used to establish the absolute solvation free-energy and enthalpy of the proton $[25,27]$. A single half-cell potential of a redox couple has not previously been measured directly in solution, and both the SHE potential and the solvation free-energy and enthalpy of the proton are conventionally assigned an arbitrary value of zero and other thermochemical values are reported relative to these standards.

Here, we model the energy that is partitioned into the translational, rotational, and vibrational modes of the products for each sequential water loss resulting from EC by many different large hydrated metal ions and obtain theoretical product ion distributions. The calculated product ion distributions accurately account for the distribution of the experimentally measured product ion abundances. These results indicate that the breadth of the energy deposited into the hydrated ions upon EC is exceedingly narrow, and that the width of the measured product ion distribution is almost entirely attributable to energy partitioning into the products during dissociation.

\section{Experimental}

Experiments were performed on a $2.75 \mathrm{~T}$ Fourier transform ion cyclotron resonance (FT/ICR) mass spectrometer [31] equipped with an ion cell that is cooled to 133 $\mathrm{K}$ using a controlled flow of $\mathrm{N}_{2}(l)$ [32]. Hydrated ions are formed from aqueous solutions of $\sim 1$ to $10 \mathrm{mM}$ of the metal ion of interest using an external nanoelectrospray ionization source [31]. The nanospray emitters are produced from borosilicate capillaries pulled to an inner tip diameter of $\sim 1-2 \mu \mathrm{m}$. A potential of +500 to $+700 \mathrm{~V}$ (relative to the $\sim 80-100{ }^{\circ} \mathrm{C}$ heated capillary entrance to the mass spectrometer) is applied to a platinum wire that is in contact with the solution. Ions are introduced into the cell of the instrument through five stages of differential pumping and are accumulated for 4-6 s. During this ion accumulation time, dry $\mathrm{N}_{2}(\mathrm{~g})$ is introduced into the vacuum chamber to a pressure of $\sim 10^{-6}$ Torr to enhance trapping and thermalization of the ions. At the end of ion accumulation, a mechanical shutter is closed to prevent additional ions from entering the cell. A 4-7 s delay before ion isolation ensures that the pressure returns to $<10^{-8}$ Torr and that the ions achieve a steady-state distribution of internal energies before EC experiments. Precursor ions were isolated and after $50 \mathrm{~ms}$, electrons were introduced into the ion cell from a heated cathode by pulsing the cathode housing to $-1.5 \mathrm{~V}$ for $120 \mathrm{~ms}$ for the transition-metal containing nanodrops and to $-1.4 \mathrm{~V}$ for $40 \mathrm{~ms}$ for the triply charged clusters. A detailed description of the experimental setup used here has been previously reported [23, 28]. Between 500-1000 spectra were coadded to improve the signal-to-noise ratios in these experiments.

To obtain the theoretical EC product ion distributions, the distribution of energy lost to translational, rotational, and vibrational modes of the products for each sequential water molecule loss is approximated by an exponential function approaching zero at highenergy. These distributions for all water molecules lost are convolved using Mathcad 14 (Parametric Technology Co., Needham, MA, USA) by multiplying the Laplace transform of each exponential function and performing the inverse Laplace transform on this product. Fragment ion abundances were fit with Gaussian functions using Igor Pro 4.07 (WaveMetrics, Inc., Lake Oswego, OR, USA). The noise level was used to estimate the fragment ion abundances at both extremes of the distributions. Professor Jamie H. Cate (University of California-Berkeley) generously provided the $\operatorname{Ir}\left(\mathrm{NH}_{3}\right)_{6} \mathrm{Cl}_{3}$ solution used in these experiments. All other metal salts came from commercial sources.

\section{Results and Discussion}

\section{Effects of Transition-Metal Ion Identity}

Broad distributions of $\mathrm{M}^{2+}\left(\mathrm{H}_{2} \mathrm{O}\right)_{n}$ and $\mathrm{M}^{3+}\left(\mathrm{H}_{2} \mathrm{O}\right)_{n}$ can be formed via nanoelectrospray ionization and trapped in the ion cell where they are thermalized to the temperature of the copper jacket surrounding the ion cell $(133 \mathrm{~K})$ [25, 31]. Individual clusters are isolated using SWIFT waveforms and are irradiated with thermally generated electrons for electron capture (EC) experiments. The product ion mass spectra resulting from $\mathrm{EC}$ by $\mathrm{M}\left(\mathrm{H}_{2} \mathrm{O}\right)_{32}^{2+}, \mathrm{M}=\mathrm{Mn}, \mathrm{Fe}, \mathrm{Co}, \mathrm{Ni}, \mathrm{Cu}$, and $\mathrm{Zn}$, are given in Figure 1a-f, respectively. EC by these precursor ions results in reduction of the nanodrop and the loss of water molecules (Pathway I, Scheme 1).

Interestingly, $\mathrm{Mn}\left(\mathrm{H}_{2} \mathrm{O}\right)_{32}^{2+}$ dissociates via an additional pathway in which a $\mathrm{H}$ atom and water molecules are lost from the reduced precursor to form a hydrated metal hydroxide cluster (Pathway II, Scheme 1). These results indicate that $\mathrm{Mn}\left(\mathrm{H}_{2} \mathrm{O}\right)_{n}^{+}$, for $n \leq 20-32$, lose a hydrogen atom readily under these conditions. In ad- 

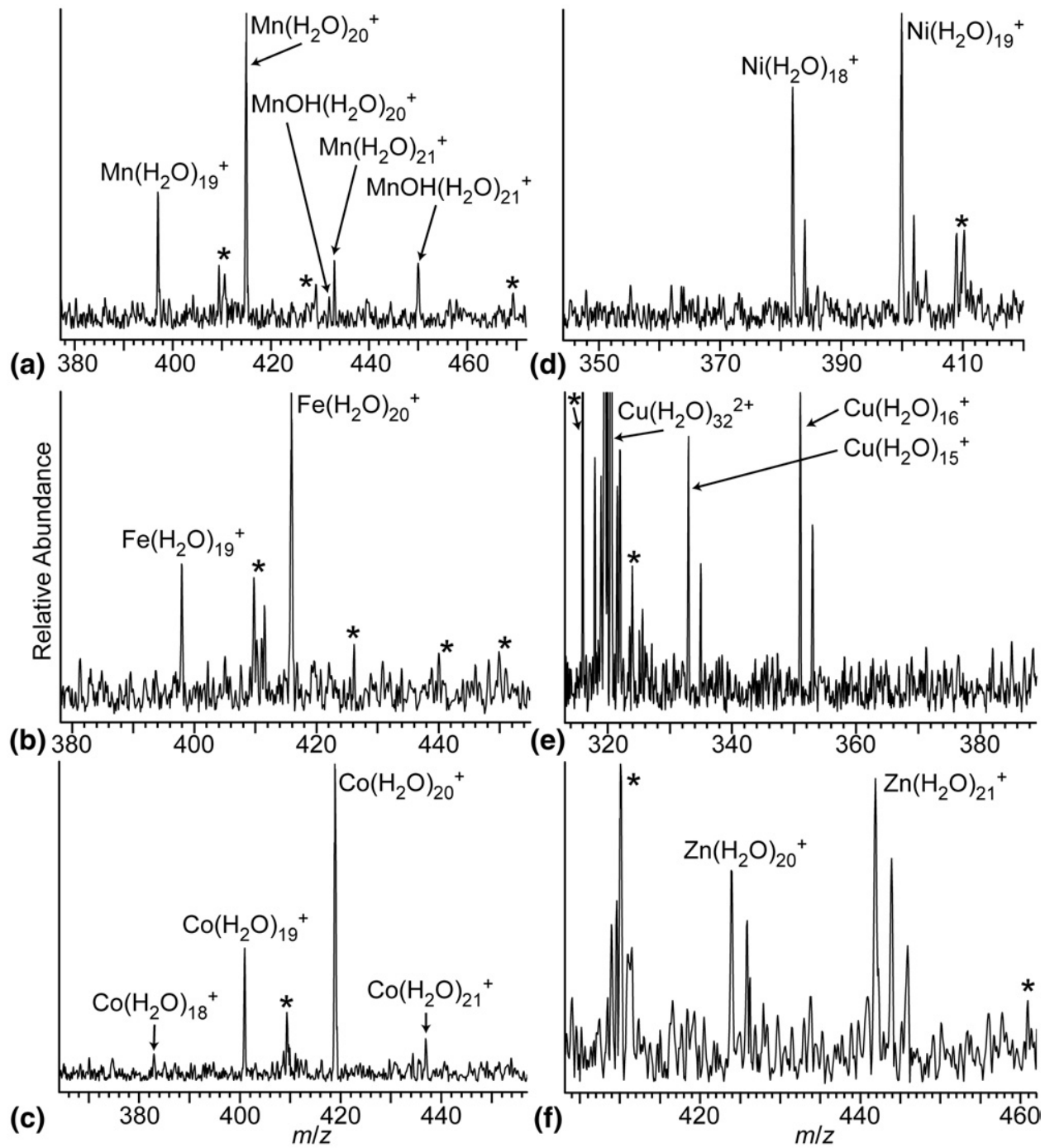

Figure 1. Electron capture dissociation product ion mass spectra of $\mathrm{M}\left(\mathrm{H}_{2} \mathrm{O}\right)_{32}^{2+}, \mathrm{M}=(\mathbf{a}) \mathrm{Mn},(\mathbf{b}) \mathrm{Fe}$, (c) $\mathrm{Co},(\mathbf{d}) \mathrm{Ni},(\mathbf{e}) \mathrm{Cu}$, and (f) $\mathrm{Zn}$. Asterisks indicate electronic noise.

dition to the water molecule loss resulting from EC, some dissociation of the precursor and fragment ions occurs as a result of the absorption of photons from the surrounding blackbody field $(<10 \%)$ during the $210 \mathrm{~ms}$ between ion isolation and detection. The average number of water molecules lost as a result of EC alone is obtained by correcting for this minor blackbody infrared radiative dissociation (BIRD) and these values are given in Table 1. The average number of water molecules lost from these clusters as a result of EC alone range from $11.3(\mathrm{M}=\mathrm{Zn})$ to $16.3(\mathrm{Cu})$. Remarkably, the widths of the product ion distributions are very narrow compared to the overall number of water molecules that are lost from the clusters upon EC (Figure 1). For

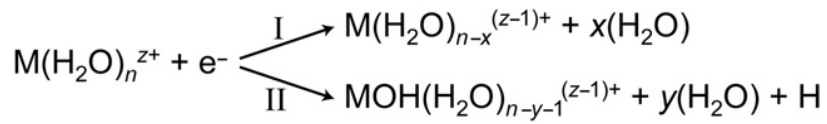

Scheme 1 example, an average of 16.3 water molecules is lost from $\mathrm{Cu}\left(\mathrm{H}_{2} \mathrm{O}\right)_{32}^{2+}$ upon $\mathrm{EC}$, but the product ion distribution is less than three water molecules wide.

By comparison, $\mathrm{M}\left(\mathrm{H}_{2} \mathrm{O}\right)_{32}^{2+}, \mathrm{M}=\mathrm{Mg}, \mathrm{Ca}, \mathrm{Sr}$ and $\mathrm{Ba}$, all lose $\sim 10.2$ water molecules upon EC and a solvent separated ion-electron pair is formed in these reduced nanodrops [21,26]. Both the greater extent of water loss, and the different number of water molecules that are lost upon $\mathrm{EC}$ of $\mathrm{M}\left(\mathrm{H}_{2} \mathrm{O}\right)_{32}^{2+}, \mathrm{M}=\mathrm{Mn}, \mathrm{Fe}, \mathrm{Co}, \mathrm{Ni}, \mathrm{Cu}$, and $\mathrm{Zn}$, indicate that the divalent transition metals are reduced in these nanodrops. Direct $\mathrm{M}^{2+}$ reduction is more favored for metals with higher second ionization energies, more stable monovalent ions, and less stable divalent ions. The second IEs of the transition metals $(15.6-20.3 \mathrm{eV})$ [33] are higher than those of the alkaline earth metals (10.0-15.0 eV) [33]. Thus, the difference in cluster reactivity for the transition metals versus the alkaline earth metals is likely a result of the higher ionization energies (IEs) of the former, although ion solvation effects also play a role. 
Table 1. Average number of water molecules lost and RE resulting from EC by $\mathrm{M}\left(\mathrm{H}_{2} \mathrm{O}\right)_{32}^{2+}$, the full-width at half-max (FWHM) of the distribution obtained by fitting the product ion abundances with Gaussian functions (Exp.) and the FWHM of the calculated kinetic energy release distribution, $P_{<x>}(E)$, for $\mathrm{M}\left(\mathrm{H}_{2} \mathrm{O}\right)_{32}^{2+}$ (Calc.)

\begin{tabular}{lcccc}
\hline $\mathrm{M}$ & $<\boldsymbol{x}>$ & RE (eV) & $\begin{array}{c}\text { Exp. } \\
\text { FWHM (eV) }\end{array}$ & $\begin{array}{c}\text { Calc. } \\
\text { FWHM (eV) }\end{array}$ \\
\hline \hline $\mathrm{Mn}$ & $12.02(2)^{\mathrm{a}}$ & $5.47(1)^{\mathrm{b}}$ & $0.58(2)^{\mathrm{c}}$ & 0.66 \\
$\mathrm{Fe}$ & $12.12(3)$ & $5.52(1)$ & $0.54(4)$ & 0.67 \\
$\mathrm{Co}$ & $12.11(2)$ & $5.51(1)$ & $0.53(3)$ & 0.67 \\
$\mathrm{Ni}$ & $13.24(1)$ & $6.11(1)$ & $0.55(8)$ & 0.75 \\
$\mathrm{Cu}$ & $16.35(2)$ & $7.83(1)$ & $0.57(3)$ & 1.01 \\
$\mathrm{Zn}$ & $11.31(5)$ & $5.11(1)$ & $0.55(6)$ & 0.61 \\
\hline
\end{tabular}

ancertainties in $\langle x\rangle$ values are propagated from the noise in the respective mass spectra.

bThe numbers in parentheses for the reported RE values correspond to a precision and not an absolute uncertainty.

'Uncertainty in exp. FWHM values are propagated from the uncertainty in the width of the best fit Gaussian function.

\section{Effects of Cluster Size on Nanodrop Reactivity}

The number of water molecules lost from the reduced nanodrops depends on cluster size. In general, both smaller and larger clusters lose fewer water molecules than intermediate size clusters. For example, EC of $\mathrm{Ca}\left(\mathrm{H}_{2} \mathrm{O}\right)_{n}^{2+}$ results in the loss of $2.8,10.2$, and 9.0 water molecules for $n=4,32$, and 47, respectively [23]. At smaller cluster sizes, fewer water molecules are lost despite high RE values because the binding energies of water molecules closest to the metal ions are higher and because more energy partitions into translational and rotational modes of the products due to the high initial effective temperatures of the reduced clusters. At much larger sizes, fewer water molecules are lost because the RE decreases due to improved ion solvation, and effects of water binding energy and energy partitioning are smaller and depend less strongly on ion size. The cluster size at which the number of water molecules reaches a maximum depends on the identity of the ion in the cluster.

In addition to the extent of water molecule loss depending on cluster size, the competition between loss of exclusively water molecules (Pathway I) and the loss of a $\mathrm{H}$ atom and water molecules (Pathway II) upon EC by $\mathrm{M}\left(\mathrm{H}_{2} \mathrm{O}\right)_{n}^{\mathrm{z}+}$ also depends on $n$. Pathway II is favored at small cluster sizes, whereas Pathway I is dominant at larger sizes, with a relatively rapid transition occurring between both pathways at $n \sim 24$ and $\sim 44$, for $\mathrm{M}=\mathrm{Ca}^{2+}[23]$, and $\mathrm{M}=\mathrm{La}^{3+}$, respectively. $\mathrm{Mn}$ is the only divalent metal ion that dissociates by both pathways with 32 water molecules (Figure 1a), although both pathways are observed for all the transition-metal ions with 24 water molecules [27].

$\mathrm{H}$ atom loss upon EC can potentially occur via several different mechanisms [26, 27]. Reduction of hydronium ion, formed transiently or facilitated by the incoming electron, would result in formation of $\mathrm{H}_{3} \mathrm{O}$, which is nominally stable in isolation [34] and should readily dissociate via $\mathrm{H}$ atom loss under these conditions. Salt-bridge structures, of the form $\mathrm{M}^{2+} \mathrm{OH}^{-} \mathrm{H}_{3} \mathrm{O}^{+}$, have been identified as stable intermediates in charge separation reactions [35]. Alternatively, a water molecule could be directly reduced to form $\mathrm{H}_{2} \mathrm{O}^{-}$, which should also dissociate via $\mathrm{H}$ atom loss. Recent ab initio methods support this latter mechanism [36]. Also, direct metal ion reduction could result in an intracluster reaction followed by $\mathrm{H}$ atom ejection, similar to the mechanism proposed for the gentle dissociation of $\mathrm{Mg}^{+}\left(\mathrm{H}_{2} \mathrm{O}\right)_{n}$ [37].

\section{Recombination Energies}

Conceivably, the recombination energy (RE) of a reduced cluster ion could be obtained from the energy corresponding to the maximum number of water molecules lost upon EC, if for each water molecule that is lost from the cluster, only the energy corresponding to the water molecule binding energy, $E_{0}$, is removed. The likelihood that no energy is partitioned into the translational and rotational modes of the products for every lost water molecule is exceedingly small when a large number of water molecules is lost. Instead, more accurate RE values can be obtained from the sum of the sequential water molecule binding energies $\left(E_{0}\right)$ and the sum of the average energy lost to the translational, rotational, and vibrational modes of the products for each sequential water molecule that is lost. Under the conditions of these experiments $\left(<10^{-8}\right.$ Torr $)$, collisional and radiative cooling should be minimal [21-23]. Clusters with fewer than 55 water molecules dissociate faster than the timescale of the experiment so kinetic shift effects are negligible [25]. Because experimentally measured sequential water molecule binding energies have not been obtained for the clusters formed in these experiments, $E_{0}$ values are obtained from a discrete implementation of the Thomson liquid drop model (TLDM) [38]. Calculated sequential water molecule binding enthalpies of $\mathrm{M}\left(\mathrm{H}_{2} \mathrm{O}\right)_{n}^{2+}$ agree with the measured binding enthalpies to within one $\mathrm{kcal} / \mathrm{mol}$ for the largest clusters for which experimental binding enthalpies have been measured $(n \leq 14)$ [38]. The values obtained from the TLDM rapidly approach the bulk heat of vaporization of water and should become increasingly accurate with increasing cluster size. Effects of ion identity are not included in the implementation of the model used here, but the charge state of the ion is taken into account. Effects of ion identity on the water molecule binding energies should not be significant for the larger cluster sizes investigated here.

To obtain the energy that is partitioned into the translational and rotational modes of the products (defined in [39] as kinetic energy release, KER), the internal energy and effective temperature of the cluster ions formed upon EC and subsequent sequential loss of water molecules are calculated assuming that the RE is instantaneously deposited into the precursor and is randomized before dissociation, which should occur for 
the larger clusters. The energy that partitions into the vibrational modes of the lost water molecules should be negligible under these conditions. Upon EC, the reduced cluster average internal energy, $U_{R}$, is given by

$$
U_{\mathrm{R}}=U_{\mathrm{P}}+\mathrm{RE}
$$

where $U_{\mathrm{P}}$ is the average internal energy of the precursor at the initial ion temperature $(133 \mathrm{~K})$. Internal energies were obtained from harmonic frequencies for a B3LYP/ $\mathrm{LACVP}^{* *}++$ energy-minimized structure of $\mathrm{Ca}\left(\mathrm{H}_{2} \mathrm{O}\right)_{14}^{2+}$, scaled by the vibrational degrees-of-freedom of the cluster of interest. The RE is given by

$$
R E=\sum E_{0}+(5 / 2) k \sum T^{*}
$$

where $\Sigma E_{0}$ is the sum of the $E_{0}$ values for each sequential water molecule that is lost and $(5 / 2) k \Sigma T^{*}$ is the sum of the energy partitioned into the products for each water molecule lost [40-43], where $k$ is the Boltzmann constant and $T^{*}$ is the effective temperature of each cluster formed as a result of each sequential water molecule loss. Although there are several models to obtain the energy partitioned into translational energy [44-46], the Klots cluster evaporation model [43] is used because it includes both translations and rotations. In this model, water molecule loss from a large water cluster results in the partitioning of $k T^{*}$ and $(3 / 2) k T^{*}$ into translational and rotational modes, respectively, for a total of $(5 / 2) k T^{*}$ for each water molecule lost. The effective temperatures of the newly formed cluster (as opposed to that of the precursor) is used for the energy partitioning values because this effective temperature should best match that of the products for dissociation through a loose transition-state with no reverse activation barrier [43]. The average internal energy of each cluster formed upon sequential water molecule evaporation is given by

$$
U_{i}\left(T_{i}^{*}\right)=U_{i+1}\left(T_{i+1}^{*}\right)-E_{0, i+1}-(5 / 2) k T_{i}^{*}
$$

where $U_{i}\left(T_{i}^{*}\right)$ and $U_{i+1}\left(T_{i+1}^{*}\right)$ are the internal energies of the newly formed cluster, $i$, and parent cluster, $i+1$, respectively, for each stepwise water molecule loss. $E_{0, i+1}$ is the binding energy of the $i+1$ cluster, and $T_{i}^{*}$ is the effective temperature of the $i$ th cluster, for each water molecule lost. Equations 1 and 2 can be combined to give the total internal energy of the reduced precursor and the internal energy of each cluster formed upon sequential water molecule loss is given by eq 3 . This results in a system of equations with more equations than unknowns ( $T^{*}$ values), which can readily be solved to obtain a unique set of $T^{*}$ values for the reduced cluster and all clusters formed by water molecule loss. From these calculated effective temperatures and the sequential $E_{0}$ values obtained from a discrete implementation of the TLDM [38], the RE is obtained using eq 2 . Because the RE values and calculated effective temperatures are obtained from modeling, and the accura- cies of these models are not well known, uncertainty in the RE values is difficult to assess. However, UV and visible laser photodissociation experiments can be used to calibrate the RE values with high accuracy by measuring the average number of water molecules lost from a cluster upon absorption of a photon of known energy. These experiments are currently underway.

The effective temperatures of select clusters after EC as a function of the number of water molecules lost, calculated using the method described above, are given in Figure 2. For $\mathrm{Ru}\left(\mathrm{NH}_{3}\right)_{6}\left(\mathrm{H}_{2} \mathrm{O}\right)_{37}^{3+}, \mathrm{EC}$ increases the cluster temperature from $\sim 130$ to $\sim 610 \mathrm{~K}$ (open circles), whereas that for $\mathrm{Ru}\left(\mathrm{NH}_{3}\right)_{6}\left(\mathrm{H}_{2} \mathrm{O}\right)_{55}^{3+}$ increases to $\sim 480 \mathrm{~K}$ (open diamonds). Sequential water molecule loss decreases the cluster internal energies and the effective temperatures close to that of the precursor before EC. The higher effective temperature for the smaller precursor cluster is due to the higher cluster internal energy owing to the fewer vibrational degrees-of-freedom and a higher RE; the RE for $\mathrm{Ru}\left(\mathrm{NH}_{3}\right)_{6}\left(\mathrm{H}_{2} \mathrm{O}\right)_{n}^{3+}$ is 8.91 and $8.53 \mathrm{eV}$ for $n=37$ and 55, respectively. The energy removed by sequential water molecule loss, $E_{\mathrm{W}}=E_{0}+$ $(5 / 2) k T_{i}^{*}$, is given on the right vertical axis in Figure 2. The differences in the $E_{W}$ values in Figure 2 are due predominantly to the different $T^{*}$ values as a result of differences in the cluster internal energies, although there are also minor differences in water molecule binding energies due to the different cluster sizes.

\section{Product Ion Distributions}

In addition to obtaining RE values, it is interesting to investigate the origin of the remarkably narrow product ion distributions resulting from EC by hydrated ions.

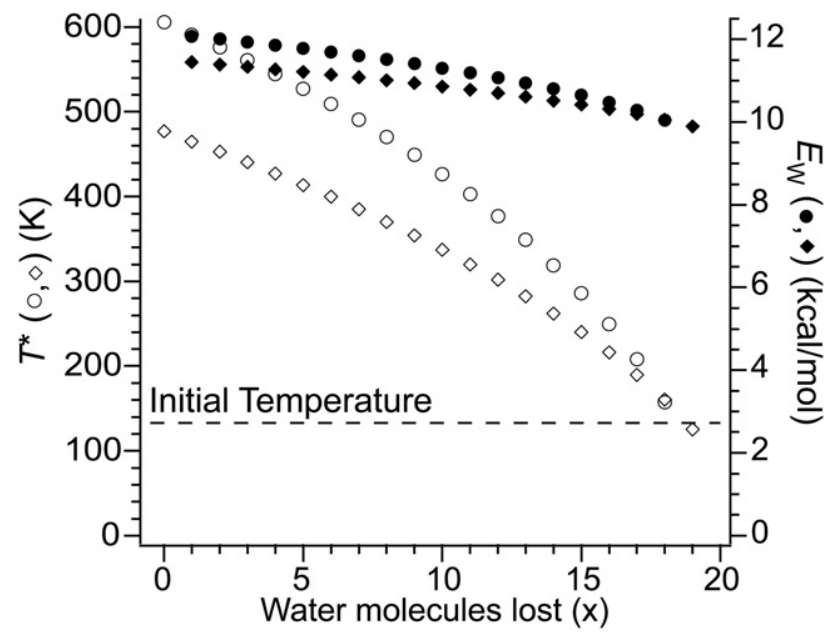

Figure 2. The calculated effective temperatures of the reduced clusters, $T^{*}$, (left vertical axis, open symbols) and the energy removed upon sequential water molecule loss from the reduced clusters, $E_{\mathrm{W}}$, (right vertical axis, closed symbols) for $\mathrm{Ru}\left(\mathrm{NH}_{3}\right)_{6}\left(\mathrm{H}_{2} \mathrm{O}\right)_{37-x}^{2+}$, circles, and $\mathrm{Ru}\left(\mathrm{NH}_{3}\right)_{6}\left(\mathrm{H}_{2} \mathrm{O}\right)_{55-x}^{2+}$, diamonds. The horizontal dashed lines indicate the initial ion temperatures (133 K). 
The observed product ion distribution should reflect broadening resulting from dissociation of the precursor and product ions as a result of the absorption of photons from the surrounding blackbody field (BIRD), the width of the energy deposited upon EC, and broadening due to the variable amount of energy removed into translational and rotational modes of the products upon each sequential water molecule loss. Because the timescale of the ion nanocalorimetry experiments for the medium to smaller sized clusters $(n \leq 65)$ are short ( $\leq 210 \mathrm{~ms}$ ), dissociation resulting from BIRD is on average 0.1 water molecules or less. Thus, broadening due to BIRD has a very minor effect on the width of the product ion distributions under these conditions.

To determine the extent of product ion broadening resulting from KER effects, the distribution of energy removed by each sequential water molecule loss must be known. The loss of a water molecule should occur through a "loose" transition-state and centrifugal effects on this barrier should be minimal. An exponential KER distribution [45], $\left.P(E)=\langle E\rangle^{-1} \exp (-E /<E\rangle\right)$, where $\left\langle E>\right.$ is the average KER value, (5/2) $k T^{*}$ [40-43], should be a good approximation for evaporation of a water molecule from a large ionic water cluster in which there is essentially no reverse activation barrier (neglecting the centrifugal barrier). The energy broadening due to the kinetic energy release distribution (KERD) of all the water molecules lost from the reduced cluster is obtained by convolving all of these distributions.

The effect of the KER for each water molecule lost on the product ion abundances is illustrated in Figure 3. The relative product ion abundances (loss of 17-19 water molecules) for EC by $\mathrm{Ru}\left(\mathrm{NH}_{3}\right)_{6}\left(\mathrm{H}_{2} \mathrm{O}\right)_{55}^{3+}$ are plotted as a function of the cumulative threshold $E_{0}$ values required to form $\mathrm{Ru}\left(\mathrm{NH}_{3}\right)_{6}\left(\mathrm{H}_{2} \mathrm{O}\right)_{55-x}^{2+}$ (bottom axis). The corresponding number of water molecules lost is given on the upper axis. The cumulative threshold energies required to form the observed product ions are substantially less than the RE (vertical dashed line) because the KER upon each sequential water molecule loss increases the energy removed by the water molecule evaporation above the threshold value, i.e., $E_{\mathrm{W}}=E_{0}+$ $(5 / 2) k T^{*}$. The KERD of the first water molecule lost, $P_{1}(E)$, is exponential and the most probable KER value is 0 for reactions with no reverse activation barrier (neglecting the centrifugal barrier). Note that the KERDs are plotted as RE $-P_{x}(E)$ to illustrate the KER effect of reducing the number of water molecules that is lost from the cluster. The convolution of the KERD for the first and second water molecule, $P_{2}(E)$, results in a most probable KER value of $\sim 2.3 \mathrm{kcal} / \mathrm{mol}$. The probability that loss of both the first and second water molecule occurs with no KER is nearly zero. Increasing the number of convoluted KER distributions to $P_{3}(E)$ and larger (select distributions shown in Figure 3) results in KERDs that are shifted to larger average values and that broaden with increasing numbers of water molecules lost. The $P_{18}(E)$ and $P_{19}(E)$ distributions correspond to the convolution of all KERDs that re-

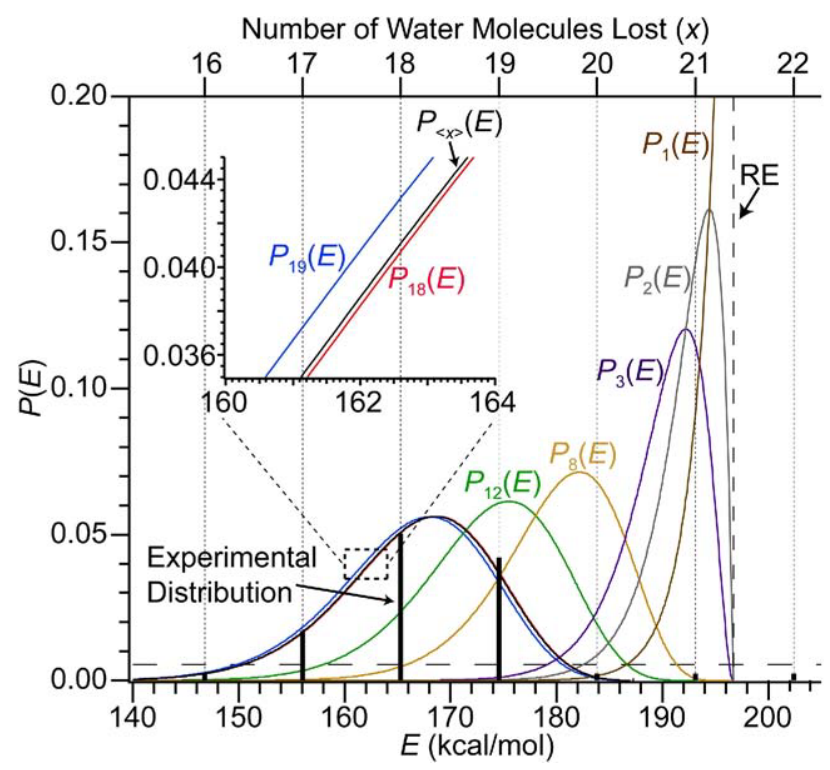

Figure 3. Experimental product ion abundances (black sticks) for EC by $\mathrm{Ru}\left(\mathrm{NH}_{3}\right)_{6}\left(\mathrm{H}_{2} \mathrm{O}\right)_{55}^{3+}$ plotted as a function of the sum of the threshold $E_{0}$ values corresponding to the formation of $\mathrm{Ru}\left(\mathrm{NH}_{3}\right)_{6}\left(\mathrm{H}_{2} \mathrm{O}\right)_{55-x}^{2+}$ (bottom horizontal axis) where $x$ is the number of water molecules lost from the reduced cluster (top axis). The cumulative translational and rotational energy release distributions, $P_{x}(E)$ s, are a convolution of translational and rotational energy release distributions for each sequential water molecule lost up to the $x$ th water molecule. The vertical dashed grey lines are a guide for the eye between the $x$ values of the upper axis and the corresponding cumulative threshold energy values on the bottom axis. The recombination energy, RE, is indicated by the black vertical dashed line. The smallest black sticks correspond to the average noise level, and the horizontal black dashed line corresponds to the average noise level plus three times the standard deviation in the noise.

sulted in the formation of these ions and are nearly identical because they differ by only the loss of the 19th water molecule, which is only a difference of roughly $\sim 1 / 18^{\text {th }}$ of the total energy deposited. To obtain the theoretical cumulative $\mathrm{KERD}, P_{<x>}(E)$, the calculated $P_{18}(E)$ and $P_{19}(E)$ are weighted by the average number of water molecules lost from the reduced cluster (18.2), i.e., $P_{<x>}(E)=(19-18.2) \cdot P_{18}(E)+(18.2-18) \cdot P_{19}(E)$. The agreement between the experimentally determined product ion distribution and that obtained theoretically is excellent, especially considering the large number of water molecules that is lost from this cluster.

To determine effects of cluster size and the magnitude of the RE value on the product ion distributions, the product ion distributions and the calculated $P_{<x>}(E)$ s for $\mathrm{Ru}\left(\mathrm{NH}_{3}\right)_{6}\left(\mathrm{H}_{2} \mathrm{O}\right)_{37}^{3+}$ and $\operatorname{Ir}\left(\mathrm{NH}_{3}\right)_{6}\left(\mathrm{H}_{2} \mathrm{O}\right)_{n}^{3+}$, $n=37$ and 55, are compared with that for $\mathrm{Ru}\left(\mathrm{NH}_{3}\right)_{6}\left(\mathrm{H}_{2} \mathrm{O}\right)_{55}^{3+}$ (Figure 4). Also included for comparison are the distributions obtained by fitting the experimentally measured product ion abundances with Gaussian functions. The full-width-at-half-maximum (FWHM) value for the theoretically calculated $P_{<x>}(E)$ and the FWHM value of the Gaussian function fit to the product ion abundances for these clusters are given in 


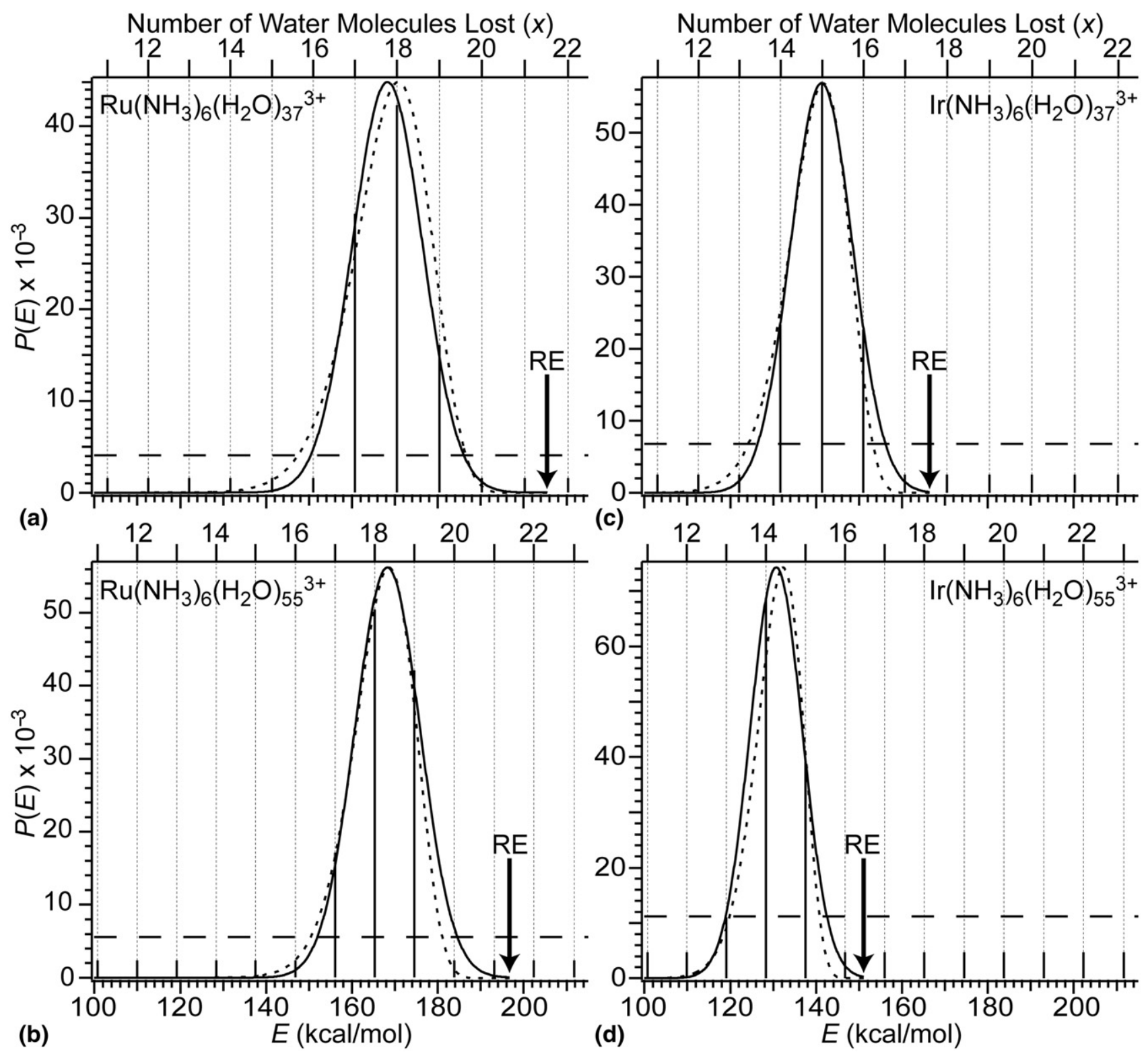

Figure 4. Experimental product ion abundances (black sticks) for $\mathrm{EC}$ by $\mathrm{M}\left(\mathrm{NH}_{3}\right)_{6}\left(\mathrm{H}_{2} \mathrm{O}\right)_{n}^{3+}$, (a) $\mathrm{M}=$ $\mathrm{Ru}, n=37$, (b) $\mathrm{M}=\mathrm{Ru}, n=55$, (c) $\mathrm{M}=\mathrm{Ir}, n=37$, and (d) $\mathrm{M}=\mathrm{Ir}, n=55$, plotted as a function of the sum of the threshold $E_{0}$ values corresponding to the formation of the given product ions (bottom horizontal axis), where $x$ is the number of water molecules lost from the reduced cluster (top axis). Calculated $P_{<x>}(E)$ distributions (black lines with the shorter dashes) and distributions obtained by fitting the product ion abundances with Gaussians (solid black lines) are compared. The vertical dashed grey lines are a guide for the eye between the $x$ values of the upper axis and the corresponding cumulative threshold energy values on the bottom axis. The smallest black sticks correspond to the average noise level and the horizontal black dashed line corresponds to the average noise level plus three times the standard deviation in the noise.

Table 2. The agreement between the theoretical and experimental distributions is excellent and indicates that product ion broadening is due almost entirely to the translational and rotational energy release into the products upon sequential water molecule evaporation. This result is consistent with the RE being a singular value for all of these ions.

The breadth of the product ion distributions are wider for the smaller clusters $(n=37)$ than for the larger clusters $(n=55)$ that contain the same ion because the former have both higher RE values and fewer internal degrees-of-freedom than the larger clusters, resulting in greater cluster internal energies and a greater KER effect. For clusters of the same size, the Ru containing clusters have wider distributions than the Ir containing clusters because the larger RE of the former clusters results in greater cluster internal energies and more KER. 
Table 2. Average number of water molecules lost and RE resulting from $\mathrm{EC}$ by $\mathrm{M}\left(\mathrm{NH}_{3}\right)_{6}\left(\mathrm{H}_{2} \mathrm{O}\right)_{\mathrm{n}}^{3+}$, the full-width at halfmax (FWHM) of the distribution obtained by fitting the product ion abundances with Gaussian functions (Exp.) and the FWHM of the calculated kinetic energy release distribution, $P_{<x>}(E)$, for $\mathrm{M}\left(\mathrm{NH}_{3}\right)_{6}\left(\mathrm{H}_{2} \mathrm{O}\right)_{\mathrm{n}}^{3+}$ (Calc.)

\begin{tabular}{lccccc}
\hline $\mathrm{M}$ & $n$ & $<x>$ & RE $(\mathrm{eV})$ & $\begin{array}{c}\text { Exp. } \\
\text { FWHM (eV) }\end{array}$ & $\begin{array}{c}\text { Calc. } \\
\text { FWHM (eV) }\end{array}$ \\
\hline \hline $\mathrm{Ru}$ & 37 & $17.78(1)^{\mathrm{a}}$ & $8.91(1)^{\mathrm{b}}$ & $0.83(2)^{\mathrm{c}}$ & 0.91 \\
$\mathrm{Ru}$ & 55 & $18.15(2)$ & $8.53(1)$ & $0.78(4)$ & 0.72 \\
$\mathrm{Ir}$ & 37 & $14.82(2)$ & $7.22(1)$ & $0.74(1)$ & 0.71 \\
$\mathrm{Ir}$ & 55 & $14.27(2)$ & $6.55(1)$ & $0.62(4)$ & 0.54 \\
\hline
\end{tabular}

ancertainties in $\langle\boldsymbol{x}\rangle$ values are propagated from the noise in the respective mass spectra.

bThe numbers in parentheses for the reported RE values correspond to a precision and not an absolute uncertainty.

'Uncertainty in exp. FWHM values are propagated from the uncertainty in the width of the best fit Gaussian function.

\section{Effect of Recombination Energy}

To investigate the effect of the RE value on the rate of energy deposition, product ion distributions for EC by $\mathrm{M}\left(\mathrm{H}_{2} \mathrm{O}\right)_{32}^{2+}, \mathrm{M}=\mathrm{Mn}, \mathrm{Ni}, \mathrm{Fe}, \mathrm{Cu}, \mathrm{Co}$, and $\mathrm{Zn}$, are compared with the calculated KER distributions assuming that the RE is deposited instantaneously (Figure 5). The FWHM values for the calculated distributions and the distributions obtained by fitting the experimental product ion distributions with Gaussians are given in Table 1. For ions with the lowest RE values $(\mathrm{M}=\mathrm{Mn}$, $\mathrm{Fe}, \mathrm{Co}, \mathrm{Ni}$, and $\mathrm{Zn}$ ), the calculated product ion distributions are slightly wider than, but in relatively good agreement with the experimental product ion distributions. This indicates that rate of energy deposition is fast compared with the rate of water evaporation for these ions. For $\mathrm{M}=\mathrm{Cu}$, which has the highest $\mathrm{RE}$ value, the calculated width is much broader than the observed product ion width. This indicates that the initial calculated temperature is too high. This discrepancy between the calculated and measured product ion distribution could occur if some water molecule loss occurred on the timescale in which the recombination energy is converted to internal modes of the precursor ion. Sequential water molecule loss would occur at lower effective temperatures and KER effects on the width of the product ion distribution would be reduced. This may be a result of a multistep electronic-to-vibrational energy conversion process or due to the formation of a relatively long-lived ion-electron pair [26] in which the metal ion is not immediately reduced and the electron is near the surface of the nanodrop. To estimate the rate of water molecule evaporation, the Klots cluster evaporation model [47] was used to calculate a lifetime of roughly $2 \times 10^{-14} \mathrm{~s}$ for reduced $\mathrm{Cu}\left(\mathrm{H}_{2} \mathrm{O}\right)_{32}^{2+}$ [22]. This lifetime is shorter than that calculated for $\mathrm{Zn}\left(\mathrm{H}_{2} \mathrm{O}\right)_{32}^{2+}$ $\left(2 \times 10^{-13} \mathrm{~s}\right)$, and may be sufficiently short that water molecules could evaporate before the full internalization of the recombination energy. These results indicate that for a fixed number of internal degrees-of-freedom, higher REs result in water evaporation rates that are more competitive with the energy deposition rates. For $\mathrm{Cu}\left(\mathrm{H}_{2} \mathrm{O}\right)_{n}^{2+}, n=24,32$, and 41 , the differences in the calculated and experimental widths are $0.5,0.4$, and 0.2 $\mathrm{eV}$, respectively. These data indicate that the rate of water molecule evaporation becomes slower than the rate of energy deposition with increasing cluster size and decreasing RE values.

\section{Effect of Cluster Reactivity}

EC by $\mathrm{Ni}\left(\mathrm{H}_{2} \mathrm{O}\right)_{24}^{2+}$ results in dissociation via Pathways I and II and the average number of water molecules lost via each pathway is 13.1 and 10.3 , respectively. The difference in the number of water molecules lost via each pathway corresponds to the energy removed upon $\mathrm{H}$ atom loss for the Pathway II process. The energy difference obtained by modeling these two processes is $1.6 \mathrm{eV}$, which corresponds to the $\mathrm{H}$ atom affinity of $\mathrm{NiOH}\left(\mathrm{H}_{2} \mathrm{O}\right)_{23}^{2+}$ [27]. The width of the experimentally measured Pathway II product ion distribution is in excellent agreement with the width of the calculated KERD (Figure 6a), indicating that the broadening of this distribution is attributable almost entirely to KER effects and that internalization of the RE is fast relative to ion dissociation. In contrast, upon dissociation of this same ion to form Pathway I products, the experimentally measured product ion distribution is significantly narrower than the calculated distribution (Figure $6 \mathrm{~b}$ ). As was the case for $\mathrm{Cu}\left(\mathrm{H}_{2} \mathrm{O}\right)_{32}^{2+}$, the discrepancy between the calculated and experimental distributions for Pathway I can be attributed to the rate of initial water molecule loss being comparable to, or faster than, that of internal energy conversion. If some water loss occurs before full internal energy conversion, the calculated effective temperature of the reduced cluster will be too high, which accounts for the wider calculated distribution. The measured product ion distribution for Pathway II is wider than that for Pathway I. This difference can be attributed to the loss of a $\mathrm{H}$ atom occurring before significant water loss. Significantly more energy is removed from the cluster when a $\mathrm{H}$ atom is lost (1.6 $\mathrm{eV})$ compared with when a single water molecule is lost $(0.5 \mathrm{eV})$. Loss of the $\mathrm{H}$ atom reduces the calculated cluster effective temperature by $\sim 120 \mathrm{~K}$, whereas the loss of a single water molecule reduces the cluster by only $\sim 20 \mathrm{~K}$. Thus, $\mathrm{H}$ atom loss in Pathway II more efficiently reduces the effective temperature than water molecule loss. At this lower temperature, the rate of water loss should be relatively slow compared with internal conversion, so that the calculated distribution fits the experimental distribution for products formed by this pathway.

\section{Conclusions}

Ion nanocalorimetry can be used to precisely characterize the internal energy deposited into activated ions, even for highly exothermic ion-electron recombination reactions. The product ion distributions resulting from 

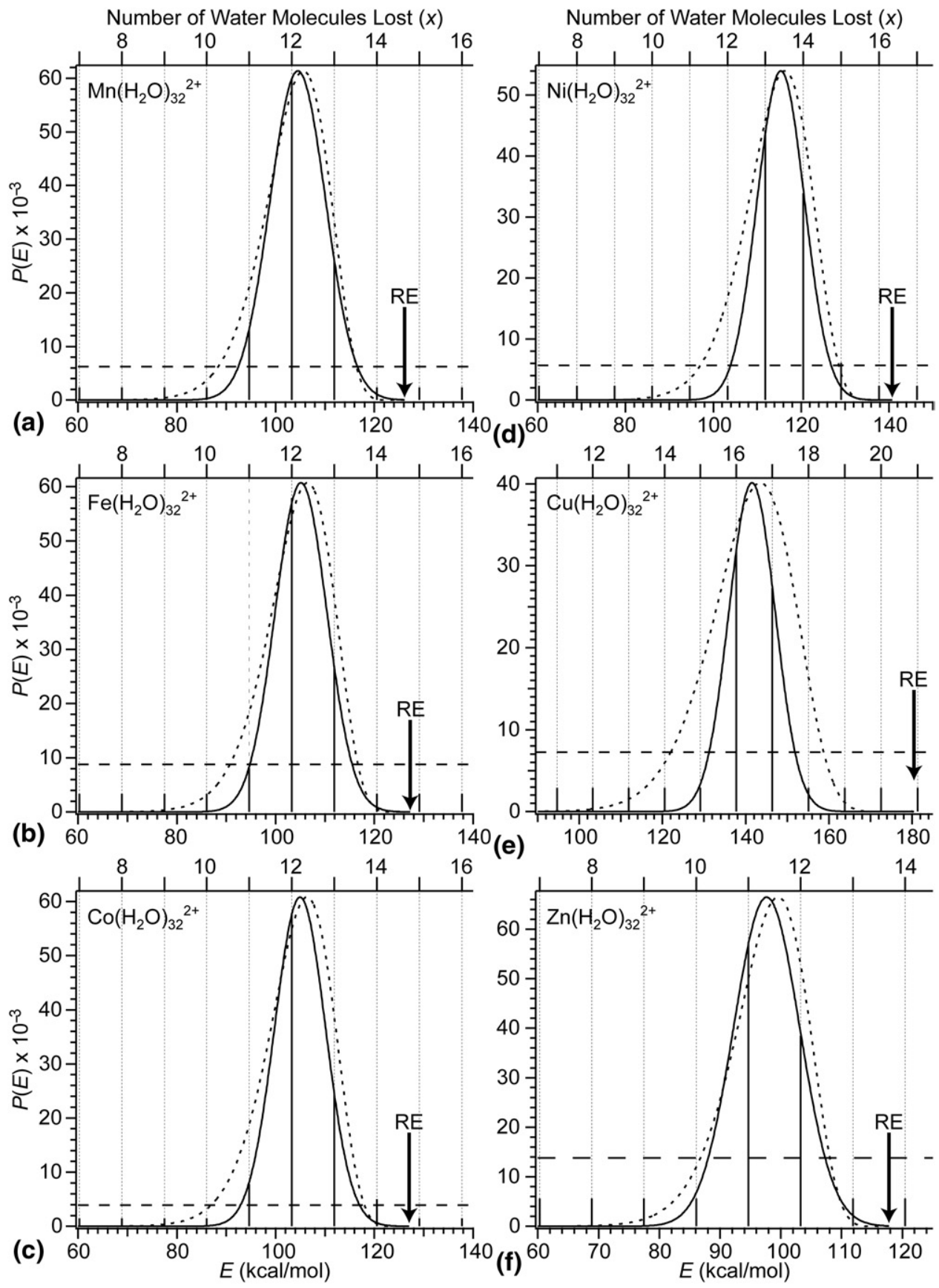

Figure 5. Experimentally measured product ion abundances for electron capture by $\mathrm{M}\left(\mathrm{H}_{2} \mathrm{O}\right)_{32}^{2+}, \mathrm{M}=$ (a) $\mathrm{Mn}$, (b) $\mathrm{Fe}$, (c) $\mathrm{Co}$, (d) $\mathrm{Ni},(\mathbf{e}) \mathrm{Cu}$, and (f) $\mathrm{Zn}$, plotted as a function of the sum of the threshold $E_{0}$ values corresponding to the formation of the given product ions (bottom horizontal axis), where $x$ is the number of water molecules lost from the reduced cluster (top axis). Calculated $P_{<x>}(E)$ distributions (black lines with the shorter dashes) and distributions obtained by fitting the product ion abundances with Gaussians (solid black lines) are compared. The vertical dashed grey lines are a guide for the eye between the $x$ values of the upper axis and the corresponding cumulative threshold energy values on the bottom axis. The smallest black sticks correspond to the average noise level, and the horizontal black dashed line corresponds to the average noise level plus three times the standard deviation in the noise.

electron capture by large hydrated ions are very narrow, and the width of these distributions can be accounted for by a statistical model to determine energy partitioning into translational and rotational modes of the products. These results indicate that the recombination energy is a singular value for these ions and that the vast majority of the recombination energy is randomized before fragmentation for the larger clusters. 


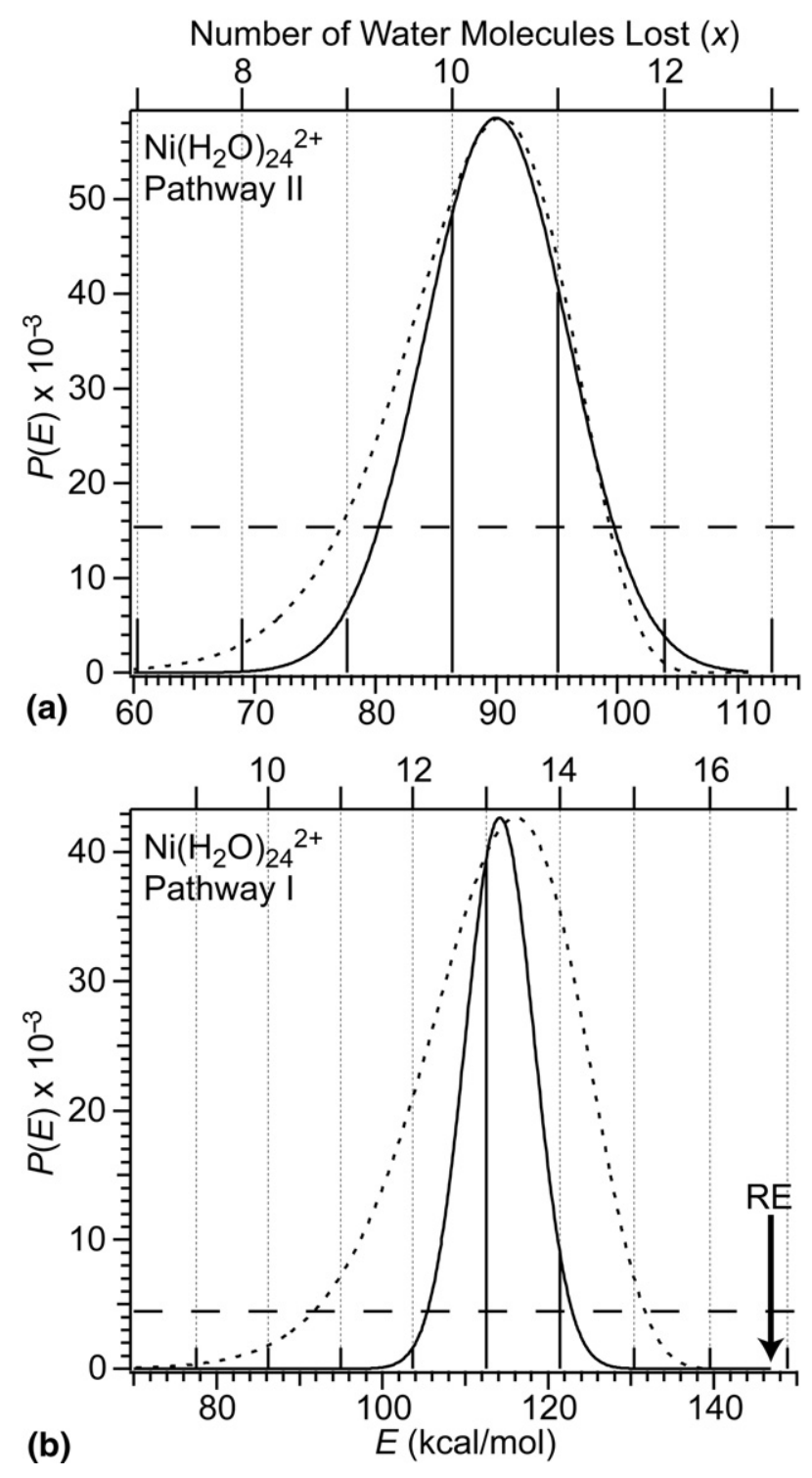

Figure 6. Experimental product ion abundances for EC by $\mathrm{Ni}\left(\mathrm{H}_{2} \mathrm{O}\right)_{24}^{2+}$ resulting in (a) loss of a $\mathrm{H}$ atom and water molecules (Pathway II) or (b) loss of exclusively water molecules (Pathway I) plotted as a function of the sum of the threshold $E_{0}$ values corresponding to the formation of the given product ions (bottom horizontal axis), where $x$ is the number of water molecules lost from the reduced cluster (top axis). Calculated $P_{<x>}(E)$ distributions (black lines with the shorter dashes) and distributions obtained by fitting the product ion abundances with Gaussians (solid black lines) are compared. The vertical dashed grey lines are a guide for the eye between the $x$ values of the upper axis and the corresponding cumulative threshold energy values on the bottom axis. The smallest black sticks correspond to the average noise level, and the horizontal black dashed line corresponds to the average noise level plus three times the standard deviation in the noise.

For some clusters where the recombination energy is high and/or the cluster size is small, water loss is competitive with energy randomization. This could be a result of a multi-step process, such as ion-electron pair formation in the droplet before reduction of the ion, or inefficient conversion of electronic-to-vibrational energy- transfer. These results provide additional support for the accuracy with which condensed phase thermochemical values, which are difficult or impossible to measure directly in solution, can be obtained from gaseous nanocalorimetry data [22, 25, 27].

This ion nanocalorimetry method should be generally applicable to measuring the extent of internal energy deposition in a broad range of activation methods. The energy deposition could be experimentally calibrated using laser-generated photons of known energy. In combination with the energy partitioning model used here, water binding energies in clusters could be obtained for much larger clusters than have been investigated previously. These values could provide information about the accuracy of the Thomson liquid drop model in which bulk physical parameters are used to deduce thermochemical information about finite size clusters.

\section{Acknowledgments}

The authors thank the National Science Foundation (CHE0718790) for generous financial support, Professor Jamie H. Cate for graciously providing $\operatorname{Ir}\left(\mathrm{NH}_{3}\right)_{6} \mathrm{Cl}_{3}$, Maria Demireva for carefully proofreading this work, and Ryan D. Leib, Jeremy T. O'Brien, Matthew F. Bush, and James S. Prell for useful discussions. The authors acknowledge the donors of the American Chemical Society Petroleum Research Fund (47916-AC6) for support of this research.

\section{References}

1. Zubarev, R. A.; Kelleher, N. L.; McLafferty, F. W. Electron Capture Dissociation of Multiply Charged Protein Cations. A Nonergodic Process. J. Am. Chem. Soc. 1998, 120, 3265-3266.

2. Breuker, K.; Oh, H. B.; Horn, D. M.; Cerda, B. A.; McLafferty, F. W. Detailed Unfolding and Folding of Gaseous Ubiquitin Ions Characterized by Electron Capture Dissociation. J. Am. Chem. Soc. 2002, 124, 6407-6420.

3. Zubarev, R. A.; Horn, D. M.; Fridriksson, E. K.; Kelleher, N. L.; Kruger, N. A.; Lewis, M. A.; Carpenter, B. K.; McLafferty, F. W. Electron Capture Dissociation for Structural Characterization of Multiply Charged Protein Cations. Anal. Chem. 2000, 72, 563-573.

4. Sze, S. K.; Ge, Y.; Oh, H. B.; McLafferty, F. W. Top-Down Mass Spectrometry of a 29-kDa Protein for Characterization of Any Posttranslational Modification to Within One Residue. Proc. Natl. Acad. Sci. U.S.A. 2002, 99, 1774-1779.

5. Mirgorodskaya, E.; Roepstorff, P.; Zubarev, R. A. Localization of O-Glycosylation Sites in Peptides by Electron Capture Dissociation in a Fourier Transform Mass Spectrometer. Anal. Chem. 1999, 71, 4431-4436.

6. Oh, H. B.; Breuker, K.; Sze, S. K.; Ge, Y.; Carpenter, B. K.; McLafferty, F. W. Secondary and Tertiary Structures of Gaseous Protein Ions Characterized by Electron Capture Dissociation Mass Spectrometry and Photofragment Spectroscopy. Proc. Natl. Acad. Sci. U.S.A. 2002, 99, $15863-15868$.

7. Robinson, E. W.; Leib, R. D.; Williams, E. R. The Role of Conformation on Electron Capture Dissociation of Ubiquitin. J. Am. Soc. Mass Spectrom. 2006, 17, 1469-1479.

8. Syka, J. E. P.; Coon, J. J.; Schroeder, M. J.; Shabanowitz, J.; Hunt, D. F. Peptide and Protein Sequence Analysis by Electron Transfer Dissociation Mass Spectrometry. Proc. Natl. Acad. Sci. U.S.A. 2004, 101, 95289533.

9. Hvelplund, P.; Liu, B.; Nielsen, S. B.; Tomita, S. Electron Capture Induced Dissociation of Peptide Dications. Int. J. Mass Spectrom. 2003, 225, 83-87.

10. Misharin, A. S.; Silivra, O. A.; Kjeldsen, F.; Zubarev, R. A. Dissociation of Peptide Ions by Fast Atom Bombardment in a Quadrupole Ion Trap. Rapid Commun. Mass Spectrom. 2005, 19, 2163-2171.

11. Zubarev, R. A.; Haselmann, K. F.; Budnik, B.; Kjeldsen, F.; Jensen, F. Towards an Understanding of the Mechanism of Electron-Capture Dissociation: A Historical Perspective and Modern Ideas. Eur. J. Mass Spectrom. 2002, 8, 337-349.

12. Iavarone, A. T.; Paech, K.; Williams, E. R. Effects of Charge State and Cationizing Agent on the Electron Capture Dissociation of a Peptide. Anal. Chem. 2004, 76, 2231-2238. 
13. Sobczyk, M.; Simons, J. Distance Dependence of Through-Bond Electron Transfer Rates in Electron-Capture and Electron-Transfer Dissociation. Int. J. Mass Spectrom. 2006, 253, 274-280.

14. Chamot-Rooke, J.; Malosse, C.; Frison, G.; Tureček, F. Electron Capture in Charge-Tagged Peptides. Evidence for the Role of Excited Electronic States. J. Am. Soc. Mass Spectrom. 2007, 18, 2146-2161.

15. Griffiths, I. W.; Mukhtar, E. S.; March, R. E.; Harris, F. M.; Beynon, J. H. Comparison of Photo-Excitation of Ions and Collisional Excitation Using Gases. Int. J. Mass Spectrom. Ion Phys. 1981, 39, 125-132.

16. Baer, T.; Dutuit, O.; Mestdagh, H.; Rolando, C. Dissociation Dynamics of $n$-Butylbenzene Ions: The Competitive Production of $\mathrm{m} / \mathrm{z} 91$ and 92 Fragment Ions. J. Phys. Chem. 1988, 92, 5674-5679.

17. Schnier, P. D.; Jurchen, J. C.; Williams, E. R. The Effective Temperature of Peptide Ions Dissociated by Sustained Off-Resonance Irradiation Collisional Activation in Fourier Transform Mass Spectrometry. J. Phys. Chem. B 1999, 103, 737-745.

18. Kenttämaa, H. I.; Cooks, R. G. Internal Energy-Distributions Acquired through Collisional Activation at Low and High-Energies. Int. J. Mass Spectrom. Ion Processes 1985, 64, 79-83.

19. Dekrey, M. J.; Kenttämaa, H. I.; Wysocki, V. H.; Cooks, R. G. Energy Deposition in $\left[\mathrm{Fe}(\mathrm{CO})_{5}\right]^{+\cdot}$ Upon Collision with a Metal Surface. Org. Mass Spectrom. 1986, 21, 193-195.

20. Wysocki, V. H.; Kenttämaa, H. I.; Cooks, R. G. Internal EnergyDistributions of Isolated Ions after Activation by Various Methods. Int. J. Mass Spectrom. Ion Processes 1987, 75, 181-208.

21. Leib, R. D.; Donald, W. A.; Bush, M. F.; O’Brien, J. T.; Williams, E. R. Internal Energy Deposition in Electron Capture Dissociation Measured Using Hydrated Divalent Metal Ions as Nanocalorimeters. J. Am. Chem. Soc. 2007, 129, 4894-4895.

22. Donald, W. A.; Leib, R. D.; O'Brien, J. T.; Bush, M. F.; Williams, E. R. Absolute Standard Hydrogen Electrode Potential Measured by Reduction of Aqueous Nanodrops in the Gas Phase. J. Am. Chem. Soc. 2008, 130, 3371-3381.

23. Leib, R. D.; Donald, W. A.; Bush, M. F.; O’Brien, J. T.; Williams, E. R. Nonergodicity in Electron Capture Dissociation Investigated Using Hydrated Ion Nanocalorimetry. J. Am. Soc. Mass Spectrom. 2007, 18, 1217-1231.

24. Leib, R. D.; Donald, W. A.; O'Brien, J. T.; Bush, M. F.; Williams, E. R. Reduction Energy of $1 \mathrm{M}$ Aqueous Ruthenium(III) Hexaammine in the Gas Phase: A Route Towards Establishing an Absolute Electrochemical Scale. I. Am. Chem. Soc. 2007, 129, 7716-7717.

25. Donald, W. A.; Leib, R. D.; Demireva, M.; O'Brien, J. T.; Prell, J. S.; Williams, E. R. Directly Relating Reduction Energies of Gaseous $\mathrm{Eu}\left(\mathrm{H}_{2} \mathrm{O}\right)_{n}^{3+}$, $n=55$ to 140, to Aqueous Solution: The Absolute SHE Potential and Real Proton Solvation Energy. J. Am. Chem. Soc. 2009, 131, 13328-13337.

26. Donald, W. A.; Leib, R. D.; O'Brien, J. T.; Holm, A. I. S.; Williams, E. R. Nanocalorimetry in Mass Spectrometry: A Route to Understanding Ion and Electron Solvation. Proc. Nat. Acad. Sci. U.S.A. 2008, 105, 1810218107

27. Donald, W. A.; Leib, R. D.; O’Brien, J. T.; Williams, E. R. Directly Relating Gas-Phase Cluster Measurements to Solution-Phase Hydrolysis, the Absolute Standard Hydrogen Electrode Potential, and the Absolute Proton Solvation Energy. Chem. Eur. J. 2009, 15, 5926-5934.

28. O'Brien, J. T.; Prell, J. S.; Holm, A. I. S.; Williams, E. R. Effects of Electron Kinetic Energy and Ion-Electron Inelastic Collisions in Electron Capture
Dissociation Measured Using Ion Nanocalorimetry. J. Am. Soc. Mass Spectrom. 2008, 19, 772-779.

29. Prell, J. S.; O’Brien, J. T.; Holm, A. I. S.; Leib, R. D.; Donald, W. A.; Williams, E. R. Electron Capture by a Hydrated Gaseous Peptide: Effects of Water on Fragmentation and Molecular Survival. J. Am. Chem. Soc. 2008, 130, 12680-12689.

30. Holm, A. I. S.; Donald, W. A.; Hvelplund, P.; Larsen, M. K.; Nielsen, S. B.; Williams, E. R. Investigation of Energy Deposited by Femtosecond Electron Transfer in Collisions Using Hydrated Ion Nanocalorimetry. J. Phys. Chem. A 2008, 112, 10721-10727.

31. Bush, M. F.; Saykally, R. J.; Williams, E. R. Formation of Hydrated Triply Charged Metal Ions from Aqueous Solutions Using Nanodrop Mass Spectrometry. Int. J. Mass Spectrom. 2006, 253, 256-262.

32. Wong, R. L.; Paech, K.; Williams, E. R. Blackbody Infrared Radiative Dissociation at Low Temperature: Hydration of $\mathrm{X}^{2+}\left(\mathrm{H}_{2} \mathrm{O}\right)_{n^{\prime}}$, for $\mathrm{X}=\mathrm{Mg}$, Ca. Int. J. Mass Spectrom. 2004, 232, 59-66.

33. CRC Handbook of Chemistry and Physics, Internet Version (accessed September, 2009), Taylor and Francis: Boca Raton, FL, 2007, http://www. hbcpnetbase.com.

34. Chen, F.; Davidson, E. R. Electronic, Structural, and Hyperfine Interaction Investigations of Rydberg Molecules: $\mathrm{NH}_{4}, \mathrm{OH}_{3}$, and $\mathrm{FH}_{2}$. J. Phys. Chem. A. 2001, 105, 10915-10921.

35. Beyer, M.; Williams, E. R.; Bondybey, V. E. Unimolecular Reactions of Dihydrated Alkaline Earth Metal Dications $\mathrm{M}^{2+}\left(\mathrm{H}_{2} \mathrm{O}\right)_{2}, \mathrm{M}=\mathrm{Be}, \mathrm{Mg}$, $\mathrm{Ca}, \mathrm{Sr}$, and Ba: Salt-Bridge Mechanism in the Proton-Transfer Reaction $\mathrm{M}^{2+}\left(\mathrm{H}_{2} \mathrm{O}\right)_{2} \rightarrow \mathrm{MOH}^{+}+\mathrm{H}_{3} \mathrm{O}^{+}$. J. Am. Chem. Soc. 1999, 121, 1565-1573.

36. Neff, D.; Simons, J. Theoretical Study of Electron Capture Dissociation of $\left[\mathrm{Mg}\left(\mathrm{H}_{2} \mathrm{O}\right)_{n}\right]^{2+}$ Clusters. Int. J. Mass Spectrom. 2008, 277, 166-174.

37. Berg, C.; Beyer, M.; Achatz, U.; Joos, S.; Niedner-Schatteburg, G.; Bondybey, V. E. Stability and Reactivity of Hydrated Magnesium Cations. Chem. Phys. 1998, 239, 379-392.

38. Donald, W. A.; Williams, E. R. Evaluation of Different Implementations of the Thomson Liquid Drop Model: Comparison to Monovalent and Divalent Cluster Ion Experimental Data. J. Phys. Chem. A 2008, 112, 3515-3522.

39. Laskin, J.; Lifshitz, C. Kinetic Energy Release Distributions in Mass Spectrometry. J. Mass Spectrom. 2001, 36, 459-478.

40. Klots, C. E. Quasi-Equilibrium Theory of Ionic Fragmentation: Further Considerations. Z. Naturforsch. Teil. A 1972, 27A, 553-561.

41. Klots, C. E. Thermochemical and Kinetic Information from Metastable Decompositions of Ions. J. Chem. Phys. 1973, 58, 5364-5367.

42. Klots, C. E. Kinetic Energy Distributions from Unimolecular Decay: Predictions of the Langevin Model. J. Chem. Phys. 1976, 64, 4269-4275.

43. Klots, C. E. Evaporative Cooling. J. Chem. Phys. 1985, 83, 5854-5860.

44. Engelking, P. C. Determination of Cluster Binding Energy from Evaporative Lifetime and Average Kinetic Energy Release: Application to $\left(\mathrm{CO}_{2}\right)_{n}^{+}$ and $\mathrm{Ar}_{n}^{+}$Clusters. J. Chem. Phys. 1987, 87, 936-940.

45. Baer, T.; Hase, W. L. Unimolecular Reaction Dynamics: Theory and Experiments; Oxford University Press: New York, 1996, pp 324-332.

46. Klots, C. E. Unimolecular Decomposition in a Spherically Symmetrical Potential. J. Chem. Phys. 1993, 98, 1110-1115.

47. Klots, C. E. Systematics of Evaporation. Z. Phys. D: At. Mol. Clusters 1991, 20, 105-109. 